\title{
On the duality of simultaneous time and size perception
}

\author{
EWART A. C. THOMAS and NANCY E. CANTOR \\ Stanford University, Stanford, California 94305
}

\begin{abstract}
When subjects are asked to judge the duration and size of visually presented circles that vary in duration and size, perceived duration is directly related to stimulus size and perceived size is, in most cases, directly related to stimulus duration. When subjects are asked to process time and size information simultaneously, their time judgments are the same as when only time processing is required, but their size judgments are less than when only size processing is required. These data are discussed within the context of an explicit model for the processing of size information, added to which is the assumption that time judgments are influenced by the time spent processing size information.
\end{abstract}

The perceived duration of a stimulus is influenced by nontemporal properties of the stimulus (see, e.g., Buffardi, 1971; Ornstein, 1969). Two recent studies (Avant, Lyman, \& Antes, 1975; Thomas \& Weaver, 1975) have attempted to account for this influence by relating perceived duration to the time spent processing the nontemporal features of the stimulus, and, in the present study, we seek to demonstrate the (convergent) validity of this approach.

The Thomas and Weaver (1975) model assumes that a visual stimulus, consisting of nontemporal information, 1 , and duration, $t$, is analyzed by a timer (t processor) and visual information processors $(\mathrm{g}$ processors), and that attention is shared among these processors. The output, $f(t, I)$, of the $f$ processor is a temporal encoding which is directly related to $t$ and to the amount of attention allocated to the timer. If I varies randomly from trial to trial, then the distribution of attention across the processors should be constant over trials, so that $f(t, I)$ is independent of $I$ and we can write it as $f(t)$. The output, $g(I, t)$, of the $\mathrm{g}$ processors contains encodings of the nontemporal stimulus features and an encoding, $g *(I, t)$, of the time spent processing $I$. Finally, it is assumed that perceived duration, $\tau$, is a weighted average,

$$
\tau=\mathrm{af}(\mathrm{t})+(1-\mathrm{a}) \mathrm{g} *(\mathrm{I}, \mathrm{t})
$$

where $a$ is directly related to the amount of attention given to the timer.

The minimal requirement for validating Equation 1 is to vary $t$ to see if $\tau$ varies in the "expected" directions. Given this requirement, there are at least three validation strategies, viz, varying both a and $\mathrm{g}^{*}$, varying $g^{*}$ but not $a$, and varying a but not $g^{*}$. The first was attempted in Thomas and Weaver (1975) by

The authors wish to thank Judith Gips for her help in running subjects. This research was supported by Grant GB-43275 from the National Science Foundation to the first author. asking subjects to memorize visually presented letters (I) in one condition but not another. It was shown that if $g^{*}$ was assumed to be independent of $t$ ( $\mathrm{t}<100 \mathrm{msec}$ ), the data were consistent with expectations derived from Equation 1. The first and second strategies will be attempted in the present study by asking subjects to judge the duration and size of circular areas of varying size (I) and duration ( $\mathrm{t}<100 \mathrm{msec})$.

In order to use Equation 1 to derive predictions for this task, it will be necessary to state a model of how size information is processed. From this model, it will be possible, incidentally, to derive some relations between perceived size and stimulus duration. Therefore, a goal of the present study is to examine the relation between perceived duration and size, and the dual relation between perceived size and duration.

\section{Processing of Size Information}

We assume that the earliest stage of processing a stimulus is an input or stimulus registration stage; the information in this stage is constant for as long as the stinulus is on and decays after the stimulus is terminated. Because the stimuli are circular areas, the size of which is relevant, we assume that the information in the input stage is spatial in nature, and it is sampled by a size scanner, which transfers the information to a size comparator. The scanner, or $g$ processor, is thought of as taking small spatial samples, one after another, and each sample is superimposed on previous samples. At any point in time. the output of the size comparator is the area of the pattern (not necessarily circular) accumulated up to that point. We refer to this area as the area $(a)$ of stimulus encoding, and we now discuss its time course with the help of Figure 1.

Clearly, a will increase as more samples are taken, and it is reasonable to suppose that $a$ increases with decreasing slope, since the greater the number of samples already taken, the more likely it is that the 


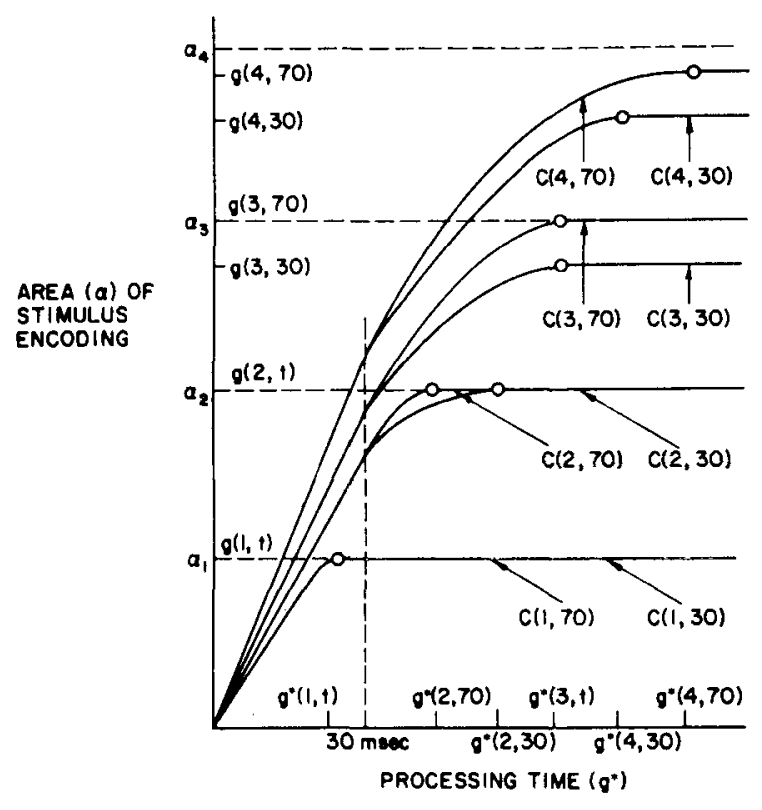

Figure 1. Accumulation of size information, a (area of stimulus encoding), over processing time, $g^{*}$, as a function of stimulus area, $A$, and stimulus duration, $t=30$ or 70 msec. $a_{i}$ is the internal representation of $A_{i}$ when $t$ is infinite. On each curve, $C(I, t)$, the open circle represents the point at which processing stops. This point determines the asymptotic size of stimulus encoding, $g(1, t)$, and the processing time, $g^{*}(I, t)$.

next sample provides information already accumulated. If the stimulus is on indefinitely, a will reach, in finite time, as asymptote, $a_{\mathrm{i}}$, which is the internal representation of the area. $A_{i}$, of the presented circle. Processing stops as soon as a reaches asymptote. When the stimulus is on for a finite time, t. the maximum value of $a$ may be less than $a_{\mathrm{i}}$; we label this maxinum value by $g(I, t)$. If the stimulus is terminated after a short time (e.g., $30 \mathrm{msec})$ and $a_{i}$ is small, a may reach asymptote before stimulus offset; further. if the stimulus duration is increased beyond this point (e.g., to $70 \mathrm{msec}$ ), the time course of $a$ is unchanged. This possibility is shown in curves $C(1,30)$ and $C(1.70)$ in Figure 1; processing time $\left(g^{*}\right)$ and area of asymptotic stimulus encoding $(\mathrm{g})$ are independent of stimulus duration. provided duration is not too short.

If $a$ is below asymptote at stimulus offset, its rate of accumulation after stimulus offset is less than if the stimulus was still on. because the information in the input stage (from which samples are taken) decays after stimulus offset. It is possible that $a_{i}$ is small enough that, for durations of 30 and $70 \mathrm{msec}$, a reaches asymptote, in which case processing time will be shorter for the longer duration. This possibility is show $n$ in curves $C(2,30)$ and $C(2,70)$ in Figure 1; area of stimulus encoding is independent of stimulus duration. Another possibility is that $a_{\mathrm{i}}$ is so large that a does not reach asymptote for the shorter stimulus duration. This is shown in curves $\mathrm{C}(3,30)$ and $\mathrm{C}(3,70)$ in Figure 1; processing time may be independent of stimulus duration, and area of stimulus encoding is greater for the longer duration. The last possibility, seen in curves $C(4,30)$ and $C(4,70)$ of Figure 1 , is where $a_{i}$ is so large that $a$ does not reach asymptote for either duration. Here processing time is longer and area of stimulus encoding is larger for the longer duration.

From this conception of information processing, the relation between $g^{*}(I, t)$ and $t$ depends on $I$. $g^{*}$ may be independent of $t$, as with asymptotes of $a_{1}$ and $a_{3}$; as $t$ increases, $g^{*}$ may decrease, as with $a_{2}$, or increase, as with $a_{4}$. The relation between $g(I, t)$ and $t$ also depends on $I$, though this relation cannot be an inverse one- $g(I, t)$ is independent of $t$ if $a_{i}$ is small and increases with $t$ if $a_{i}$ is large. Since, by assumption, time judgments are partially determined by $g^{*}$ and size judgments are determined by $g$, we can now see why effects due to stimulus duration, stimulus area, and their interaction may be seen in one set of judgments but not in another.

So far, we have not included the effects of varying stimulus-response mapping. Suppose that the subject is presented either with stimuli of area $a_{0}+\delta$ and $a_{0}+3 \delta$ (set size, $\mathrm{N}=2$ ) or with stimuli of area $a_{0}$, $a_{0}+\delta, a_{0}+2 \delta$, and $a_{0}+3 \delta(\mathrm{N}=4) .{ }^{1}$ The model presented in Figure 1 supposes that $\mathrm{g}^{*}(\mathrm{I}, \mathrm{t})$ includes processing up to the point at which the subject has sampled all useful size information. If this assumption is correct, then varying $\mathbf{N}$ should not affect processing time and perceived duration. However, it may be that $g^{*}$ is the time required to make an implicit size judgment of "small" or "large." If so, we might expect $\mathrm{g}^{*}$ to increase as $\mathrm{N}$ increases, because a mapping of two stimuli to one response probably requires more processing time than a mapping of one stimulus to one response (Kornblum, 1973).

This account of the processing of size information holds for a task in which the subject is required to make only tinie judgments. Suppose now that the subject is required to process both temporal and size information on each stimulus presentation. According to our account, the extra requirement of this mixed (time and size) condition should have no effect on time judgments, since both the $f$ and $g$ processors are already in operation in the pure-time condition. Next, suppose that the subject is required to process only size information. Then the $f$ processor is not used and the attention given to the $\mathrm{g}$ processor is increased. We assume that the effect of this increase of attention to the $\mathrm{g}$ processor is to increase the slopes of the area-time curves shown in Figure 1 by increasing the rate at which the $g$ processor samples information. Increasing the slope of a curve will generally increase the asymptotic encoding area, so we might expect that 
perceived size would be larger in the pure-size condition than in the mixed condition.

In the experiment to be reported, stimulus set size iv was varied across subjects, using the spacings between stimulus area discussed above. Each subject was presented with pure-time, pure-size, and mixed conditions; we refer to this variable as processing load, L. We denote stimulus area and stimulus duration by $A$ and $T$, respectively. The two dependent variables are perceived duration, $\tau$, obtained from the pure-time and mixed conditions, and perceived size, obtained from the pure-size and mixed conditions.

Let us consider the expected effects on $\tau$. From Equation 1,

$$
\begin{aligned}
& T(I, 70)-\tau(I, 30) \\
& =\mathbf{a}[f(70)-f(30)]+(1-a)\left[g^{*}(I, 70)-g^{*}(I, 30)\right]
\end{aligned}
$$

and

$$
\begin{aligned}
\pi\left(a_{0}+3 \delta, \mathrm{t}\right)-\pi\left(a_{0}+\delta, \mathrm{t}\right) \\
=(1-\mathrm{a})\left[\mathrm{g}^{*}\left(a_{0}+3 \delta, \mathrm{t}\right)-\mathrm{g}^{*}\left(a_{0}+\delta, \mathrm{t}\right)\right]
\end{aligned}
$$

In Figure 1, there is one case [curves $\mathrm{C}(2, \mathrm{t})$ ] where $\mathrm{g}^{*}$ decreases as $t$ increases. However, it is likely that such a decrease in $\mathrm{g}^{*}$ would be offset by the increase in $\mathrm{f}(\mathrm{t})$, and we would still expect, from Equation 2, that there would be a T main effect. Also from Figure $1, \mathrm{~g}^{*}$ tends to increase with stimulus area. $t$ constant. Therefore, from Equation 3, we would expect an A main effect. There would be no $A$ by $T$ interaction if the two values of $\mathrm{A}$ corresponded to $a_{1}$ and $a_{3}$ in Figure 1, but there would be an interaction if the values of $A$ were, e.g., $a_{3}$ and $a_{4}$. This interaction would occur because the $T$ effect increased with $\mathrm{A}$. One hypothesis, discussed above, concerning $\mathrm{N}$ led to the expectation that $\tau\left(a_{0}+\right.$ $\delta, t)$ and $\tau\left(a_{0}+3 \delta, t\right)$ should be less when $a_{0}+\delta$ and $a_{0}+3 \delta$ are the only two stimulus sizes used $(\mathrm{N}=2)$ than when sizes $a_{0}$ and $a_{0}+2 \delta$ are included $(N=4)$. That is, there should be an $\mathrm{N}$ main effect when only sizes $a_{0}+\delta$ and $a_{0}+3 \delta$ are considered. According to the other hypothesis concerning $N$, there should be no $\mathrm{N}$ main effect. Finally, there should be no main or interaction effects due to $\mathrm{L}$.

Let us turn now to the expected effects on perceived size. From Figure 1, we see that perceived size should increase with $t$, provided $a_{i}$ is large, and should increase with $a_{i}$; i.e., there should be a $T$ main effect and an $A$ main effect. There would be an $A$ by $T$ interaction if the two values of $A$ corresponded to $a_{2}$ and $a_{3}$, but not if they corresponded to $a_{3}$ and $a_{4}$. As argued earlier, there should be an $L$ main effect, perceived size being larger in the pure-size condition than in the mixed condition.

The following experiment was done to check these expectations.

\section{METHOD}

\section{Subjects}

The subjects were 24 undergraduates at Stanford University receiving credit toward introductory psychology for participation in individual sessions of about $1 \mathrm{~h}$.

\section{Stimuli}

The stimuli were red circular areas presented on a lighted background in a moditied three-field Iconix tachistoscope. The circular diameters were $7.54 .8 .33 .9 .53,10.32$. and $11.11 \mathrm{~mm}$ for Stimuli 1. 2.3,4a, and $4 \mathrm{~b}$, respectively. A stimulus was presented for 30 or $70 \mathrm{msec}$ on each trial. Two groups of subjects were used in the experiment. Twelve subjects in Group 1 were shown Stimuli 2 and $4 a$ : and 12 subjects in Group 2 saw Stimuli 1, 2, 3, and $4 b$.

\section{Responses}

Each subject was trained to discriminate between blank durations (i.e.. white visual fields with no circle) of $15 \mathrm{msec}$ ("short"), 50 msec ("medium"), and $85 \mathrm{msec}$ ("long"), On each trial, the subjects were required to categorize the duration of the circle as "short," "medium," or "long." The subjects were also trained to discriminate between the "small" stimulus circles (Stimulus 2 in Group 1 and Stimuli 1 and 2 in Group 2) and the "large" circles (Stimulus 4a in Group 1 and Stimuli 3 and $4 b$ in Group 2) presented for a constant $50 \mathrm{msec}$.

\section{Procedure}

Each subject received three types of trial blocks. In a "mixed" block, each trial consisted of a blank field presented for $500 \mathrm{msec}$ followed by a 30- or 70-msec exposure of the circle. After a further 500 -msec delay, the subject was given a response cue (" $t$ " or " $s ")$. which signaled whether a time or size judgment was required on that trial. The mixed block contained 12 " $t$ " trials and 12 "s" trials presented in a random order. Each subject also received a "pure" time block of 24 time trials and a "pure" size block of 24 size-judgment trials. Trials were presented in the pure blocks in the same nianner as in the mixed blocks. with the exception of a constant " $t$ " or " $s$ " response card cuing the response on each trial. In all blocks. the subject was asked to wait for the response cue before responding. The subject was aware that both duration and size were varied over trials. Each block contained six presentations of the small and large circles for 30 - and $70-\mathrm{msec}$ exposure durations. A different random order of presentation of stimuli was used for the three types of blocks, but the order remained constant over subjects.

The session began with 21 practice time trials with blank durations and 16 practice size trials with circles presented for $50 \mathrm{msec}$. All subjects were able to discriminate the durations and sizes of the circles by the end of the practice trials. The subjects were then tested on two mixed trial blocks and one pure time block and one pure size block. The order of the trial blocks was counterbalanced over subjects, within the constraint that the pure size and time blocks appear in the first and third positions and the mixed blocks in the second and fourth positions.

\section{RESULTS}

The temporal judgments "short," "medium," and "long" were coded 0,1 , and 2 , respectively, and, for each subject and type of trial block, pure-time or mixed. the average was found for each stimulus type. The size judgments "small" and "large" were coded 0 and 1 . respectively, and were averaged in the same manner as the temporal judgments. The time and size data were subjected to separate, parallel analyses of 


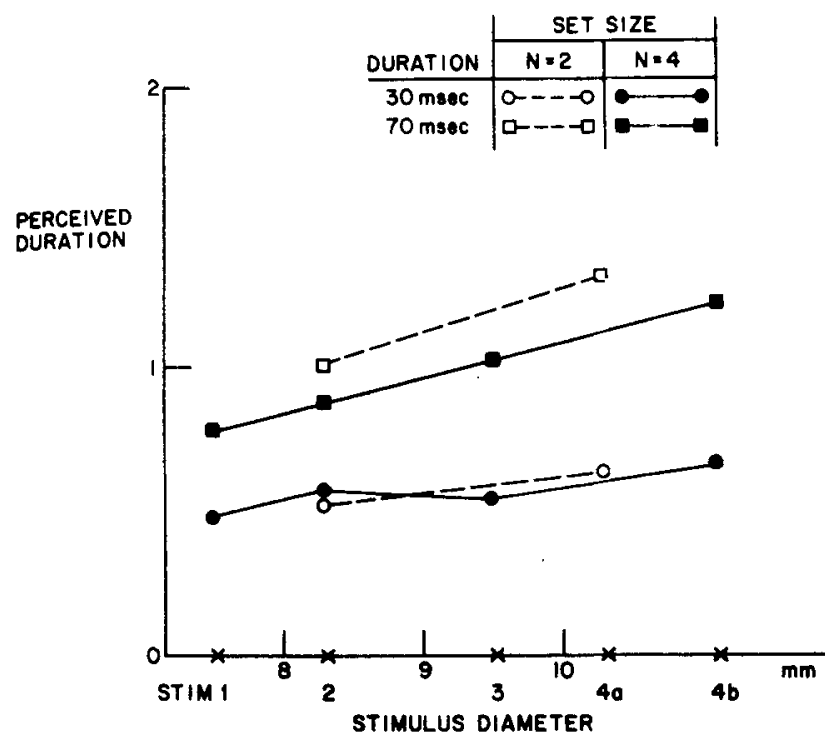

Figure 2. Temporal judgments, averaged over subjects and processing load, for each set size, stimulus duration, and stimulus area.

variance. The variables of interest are stimulus set size, $\mathrm{N}$ (the only between-subject variable), processing load, L, stimulus area, A, and stimulus duration, T.

We first consider the time data. These data were subjected to three analyses of variance; one for one between- and three within-subject variables, which omits the data for Stimuli 1 and 3 for $\mathrm{N}=4$ and involves all 24 subjects; and one three-factor analysis for each level of $\mathrm{N}$, involving 12 subjects. The three analyses yield similar results. As expected, the $\mathrm{T}$ and

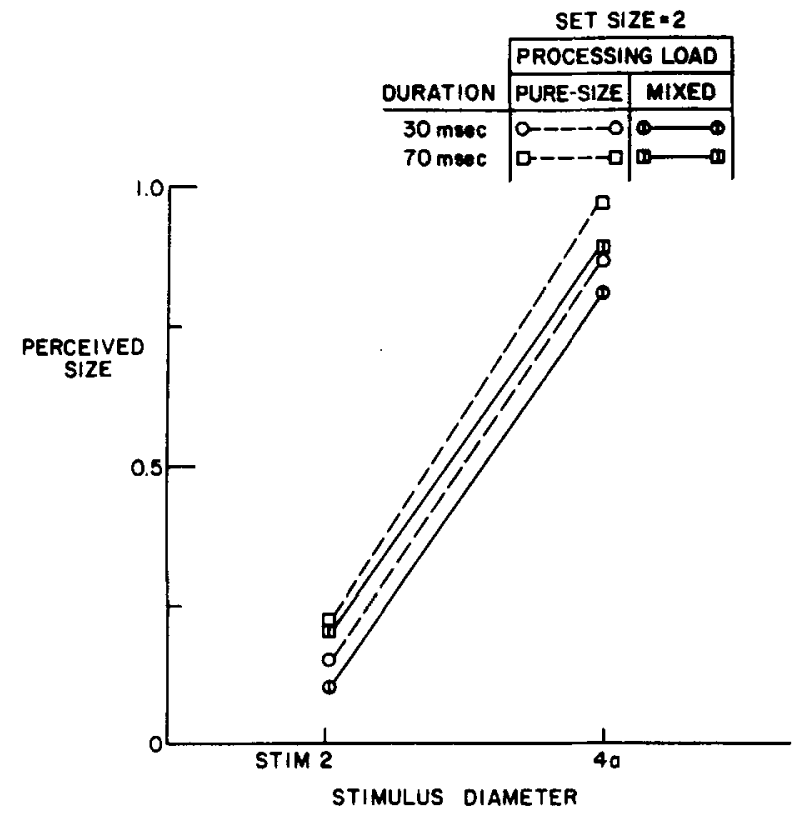

Figure 3. Size judgments, averaged over subjects, for each processing load, stimulus duration, and stimulus area; set size $=\mathbf{2}$.
A main effects are significant $(p<.001)$. From the first analysis, the $A$ by $T$ interaction is significant $[F(1,22)=6.1, p<.05]$, and it occurs because, as expected, the $\mathrm{T}$ effect increases with $\mathrm{A}$. The $\mathrm{N}$ effect is not significant, contrary to one hypothesis; and there is no significant interaction involving $\mathrm{N}$. Finally, no main or second-order interaction effect due to $\mathrm{L}$ approached statistical significance. These data, averaged across subjects and values of $L$, are presented in Figure 2.

Next we consider the size data. As expected, there is an $A$ main effect $(\mathrm{p}<.001)$. When $\mathrm{N}=2$, size judgments are significantly greater when $T=70$ than when $\mathrm{T}=30 \mathrm{msec}[\mathrm{F}(1,11)=6.4, \mathrm{p}<.05]$. When $\mathrm{N}=4$, this direct relation between perceived size and duration is seen only in the mixed condition. In the pure-size condition, there is a reversal of the expected trend $(|t|=2.3, p<.025)$. The $\mathrm{A}$ by $\mathrm{T}$ interaction is not significant. The $\mathrm{L}$ main effect was smaller than expected $[F(1,11)=2.83, \mathrm{p}<.25$, and $F(1,11)=$ $3.32, \mathrm{p}<.1$, for $\mathrm{N}=2$ and 4 , respectively]. However. an inspection of each subject's data shows that, for 17 out of 24 subjects, size judgments were smaller in the mixed condition than in the pure-size condition $(Z$, for a onetailed sign test, $=1.84, p<.05$ ). No other effects approached statistical significance. These data, averaged over subjects, are shown in Figures 3 and 4 .

\section{CONCLUDING REMARKS}

The direct relation, found in the present study, between perceived duration and stimulus area is

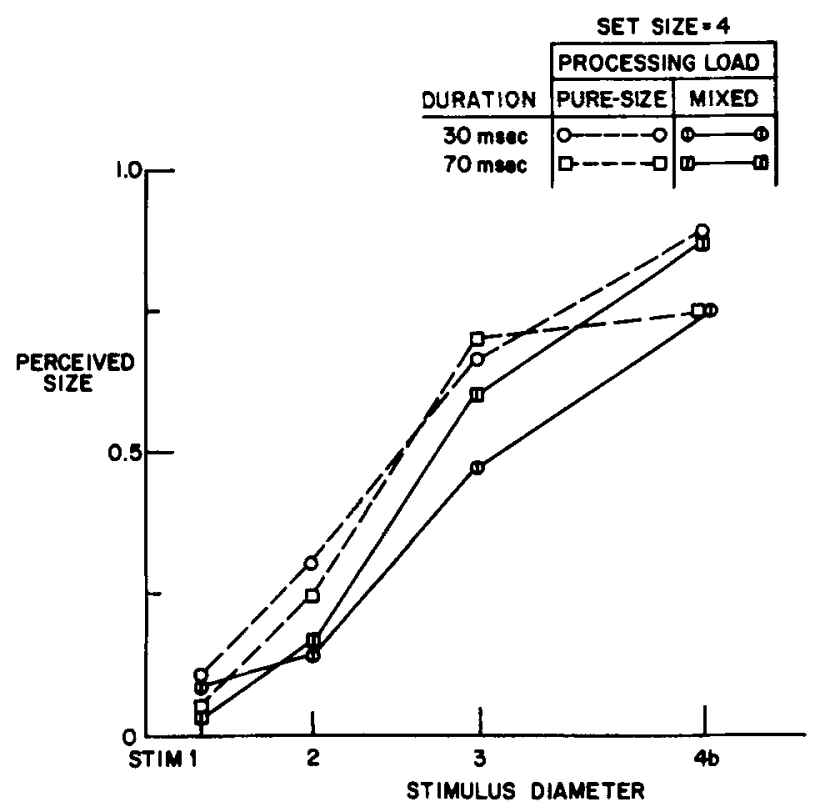

Figure 4. Size judgments, averaged over subjects, for each processing load, stimulus duration, and stimulus area; set size $=4$. 
consistent with previous results of Mo and Michalski (1972), who used unfilled circles, and Avant et al. (1975), who used random-dot matrices. This relation is influenced by stimulus duration for the range of variables used here, but it does not appear to be influenced by stimulus set size. The fact that set size has no effect in our experiment supports our assumption that the outcome of processing is an estimate, derived from sampling, of stimulus area, and that $\mathrm{g}^{*}$ does not include the extra time required to categorize the estimate as "small" or "large." The effect of stimulus area and the interaction between stimulus area and duration can be accounted for by appealing to the model, given in Figure 1, of the processing of size information.

This model also allows us to discuss the dual relation, that between perceived area and stimulus duration. For the most part, our expectation of a direct relation between these two variables has been confirmed. A related study by Mo and Jesky (1971) had subjects make discriminations between two line lengths that were presented for $.1, .5$, or $.9 \mathrm{sec}$, and found that accuracy was independent of duration when the durations appeared equally often. This independence can be accounted for by our model if we assume that the two line lengths correspond to $a_{1}$ and $a_{2}$ in Figure 1.

It may be noted that, because of our response coding, $0 \equiv$ "small," $1 \equiv$ "large," the numerical averages for size judgments represent the proportion of errors when considering "small" stimuli and the proportion of correct responses when considering "large" stimuli. Our results show that it is not the case that an increase in stimulus duration simply increases the accuracy of size judgments, because this would imply that the average numerical response to "small" stimuli would be less at the longer duration. It appears, then, that it is more useful to use "size of stimulus encoding" than "accuracy" in describing the time course of size information.

An important assumption in our theorizing is that the processors of temporal and nontemporal information are parallel and share attention (Thomas $\&$ Weaver, 1975). Some evidence for the separateness of these two types of processors comes from studies by Allan and Kristofferson (1974), Allan, Kristofferson, and Wiens (1971), and Zacks (1970). In these studies, the nontemporal information is the luminance of a stimulus, and the typical finding is that discriminable changes in luminance do not affect duration discriminations. Within a "processing time" framework for explaining time perception, one can say that changes in luminance do not affect processing time, probably because processing time is very short, owing to the peripheral position of brightness detectors. This, however, raises the question: how central does processing have to be before certain effects on temporal and nontemporal judgments can be observed? Another way of examining the central-peripheral issue is to use disjoint, rather than continuous, areas, and to vary the shape or contour of a given area. We are currently trying to answer this question.

\section{REFERENCES}

Allan, L. G., \& Kristofferson, A. B. Psychophysical theories of duration discrimination. Perception \& Psychophysics, 1974, 16. 26-34.

Allan, L. G., Kristofferson, A. B., \& Wiens, E. W. Duration discrimination of brief light flashes. Perception \& Psychophysics, 1971. 9. 327.334.

Avant, L. L., Lyman, P. J., \& Antes, J. R. Effects of stimulus familiarity on judged visual duration. Perception \& Psychophysics, 1975, 17, 253-262.

BUFFARDI.. L. Factors affecting the filled-duration illusion in the auditory, tactual and visual modalities. Perception \& Psychophysics, 1971, 10. 292-294.

Kornblum, S. Sequential effects in choice reaction time: A tutorial review. In S. Kornblum (Ed.). Attention and performance IV. New York: Academic Press, 1973.

Mo, S. S., \& Jesky, L. Temporal set associated with brief stimulus duration in a psychophysical recognition of length. Psychonomic Science, 1971, 22, 337.

Mo.S. S. . \& Michalski, U. A. Judgment of temporal duration of area as a function of stimulus configuration. Psychonomic Science, 1972, 27, 97.98.

Ornstein, R. On the experience of time. Baltimore: Penguin Books. 1969.

Thomas, E. A. C., \& Weaver, W. B. Cognitive processing and time perception. Perception \& Psychophysics, 1975, 17. 363-367.

ZACKs, J. L. Temporal summation phenomena at threshold: Their relation to visual mechanisms. Science, 1970, 170, 197-199.

\section{NOTE}

1. In order to derive the effects of varying stimulus area $\mathbf{A}_{i}$, it is more convenient to state our arguments in terms of subjective area $a_{i}$, since this facilitates reference to Figure 1 . It is assumed that there is a $1-1$ correspondence between $A_{i}$ and $a_{i}$.

(Received for publication December 21, 1974; revision received April 11, 1975.) 